\title{
ON THE STABILITY OF THE LOCALIZED SINGLE-VALUED EXTENSION PROPERTY UNDER COMMUTING PERTURBATIONS
}

\author{
PIETRO AIENA AND MICHAEL M. NEUMANN \\ (Communicated by Thomas Schlumprecht)
}

\begin{abstract}
This article concerns the permanence of the single-valued extension property at a point under suitable perturbations. While this property is, in general, not preserved under sums and products of commuting operators, we obtain positive results in the case of commuting perturbations that are quasi-nilpotent, algebraic, or Riesz operators.
\end{abstract}

\section{INTRODUCTION AND BASIC DEFINITIONS}

The single-valued extension property (SVEP) dates back to the early days of local spectral theory and appeared first in the work of Dunford [14], 15]. A thorough discussion of this property within the theory of spectral and generalized spectral operators may be found in the seminal monographs by Dunford-Schwartz [16] and by Colojoară and Foiaş 12. The following localized version of SVEP was introduced by Finch [17]. As witness by the more recent accounts in [1] and 21, SVEP has now developed into one of the major tools in the local spectral theory and Fredholm theory for operators on Banach spaces.

To fix notation, throughout this article let $X$ be a non-zero complex Banach space, and let $L(X)$ denote the Banach algebra of all bounded linear operators on $X$. As usual, given $T \in L(X)$, let $\operatorname{ker} T$ and $R(T)$ stand for the kernel and range of $T$. Also, the spectrum, resolvent set, and the spectral radius of $T$ are denoted by $\sigma(T), \rho(T)$, and $r(T)$, respectively.

Definition 1.1. An operator $T \in L(X)$ is said to have the single-valued extension property at a point $\lambda \in \mathbf{C}$ (for brevity, SVEP at $\lambda$ ), provided that, for every open disc $D \subseteq \mathbb{C}$ centered at $\lambda$, the only analytic function $f: D \rightarrow X$ that satisfies

$$
(\mu I-T) f(\mu)=0 \text { for all } \mu \in D
$$

is the function $f \equiv 0$ on $D$. Moreover, $T$ is said to have the SVEP if $T$ has the SVEP at every point $\lambda \in \mathbf{C}$.

Evidently, an operator $T$ has SVEP at a point $\lambda$ precisely when $\lambda I-T$ has SVEP at 0 . Moreover, SVEP at a point is inherited by restrictions to closed invariant subspaces.

Received by the editors April 22, 2011 and, in revised form, September 26, 2011.

2010 Mathematics Subject Classification. Primary 47A10, 47A11; Secondary 47A53, 47A55.

Key words and phrases. Localized single-valued extension property, quasi-nilpotent part, analytic core, Kato decomposition and quasi-Fredholm operators, semi-Browder operators and Riesz operators. 
It turns out that the localized SVEP is intimately related to certain conditions from classical operator theory. Let $p(T)$ denote the ascent of an operator $T \in L(X)$, i.e., $p(T)$ is the smallest non-negative integer $p$ for which $\operatorname{ker} T^{p}=\operatorname{ker} T^{p+1}$, if such an integer exists, and otherwise $p(T)=\infty$. Analogously, let $q(T)$ be the descent of an operator $T$; i.e., $q(T)$ is the smallest non-negative integer $q$ for which $R^{q}(T)=R^{q+1}(T)$ if such an integer exists, and otherwise $q(T)=\infty$. Note that

$$
p(\lambda I-T)<\infty \Rightarrow T \text { has SVEP at } \lambda,
$$

while dually, for the adjoint operator $T^{*} \in L\left(X^{*}\right)$, we have

$$
q(\lambda I-T)<\infty \Rightarrow T^{*} \text { has SVEP at } \lambda ;
$$

see [1, Theorem 3.8]. Also, let $\sigma_{\mathrm{a}}(T)$ and $\sigma_{\mathrm{s}}(T)$ denote the approximate point spectrum and the surjectivity spectrum of $T$, respectively; see 1 . From the definition of the localized SVEP and the identity $\sigma_{\mathrm{a}}\left(T^{*}\right)=\sigma_{\mathrm{s}}(T)$ it is immediate that

$$
\sigma_{\mathrm{a}}(T) \text { does not cluster at } \lambda \Rightarrow T \text { has SVEP at } \lambda \text {, }
$$

and dually

$$
\sigma_{\mathrm{s}}(T) \text { does not cluster at } \lambda \Rightarrow T^{*} \text { has SVEP at } \lambda \text {. }
$$

The localized SVEP may also be described in terms of certain subspaces that naturally arise in local spectral theory. Indeed, let $\rho_{T}(x)$ be the so-called local resolvent set of $T$ at the point $x \in X$, defined as the union of all open subsets $U$ of C for which there exists an analytic function $f: U \rightarrow X$ with the property that

$$
(\lambda I-T) f(\lambda)=x \quad \text { for all } \lambda \in U .
$$

The local spectrum $\sigma_{T}(x)$ of $T$ at $x$ is the set defined by $\sigma_{T}(x):=\mathbf{C} \backslash \rho_{T}(x)$. Obviously, $\sigma_{T}(x)$ is a closed subset of $\sigma(T)$.

Any analytic function $f$ that satisfies (4) may be viewed as a local extension of the resolvent function of $T$, since $f(\lambda)=(\lambda I-T)^{-1} x$ for all $\lambda \in \rho(T) \cap U$. In general, the analytic solutions of (4) are not uniquely determined. However, if $T$ has SVEP at $\lambda$, then any two analytic solutions of (4) coincide in an open disc centered at $\lambda$.

For every subset $F$ of $\mathbf{C}$, the analytic spectral subspace of $T$ associated with $F$ is the set $X_{T}(F):=\left\{x \in X: \sigma_{T}(x) \subseteq F\right\}$. It is easy to see from the definition that $X_{T}(F)$ is a $T$-hyperinvariant linear subspace of $X$ and that $X_{T}\left(F_{1}\right) \subseteq X_{T}\left(F_{2}\right)$ whenever $F_{1} \subseteq F_{2}$. Even for a closed set $F \subseteq \mathbb{C}$, the space $X_{T}(F)$ need not be closed. Also, it should be noted that, by [21, Proposition 1.2.16],

$$
T \text { has } \mathrm{SVEP} \Leftrightarrow X_{T}(\emptyset)=\{0\} \Leftrightarrow X_{T}(\emptyset) \text { is closed. }
$$

Next we introduce two invariant subspaces which are of particular importance in this article. The investigation of these subspaces dates back to Mbekhta 22 . and Vrbová [27, while the intimate connection to the localized SVEP was pursued further in [6], 7], and [8]; see also [1] for a detailed discussion.

Definition 1.2. The quasi-nilpotent part of an operator $T \in L(X)$ is the set

$$
H_{0}(T):=\left\{x \in X: \lim _{n \rightarrow \infty}\left\|T^{n} x\right\|^{\frac{1}{n}}=0\right\},
$$

while the analytic core of $T$ is the set $K(T):=X_{T}(\mathbb{C} \backslash\{0\})$. 
Both spaces are $T$-hyperinvariant linear subspaces of $X$ and fail to be closed in general. Moreover, by [1, Theorems 1.21, 1.22, and 2.18], we always have $T(K(T))=K(T)$, and the identity $K(T)=X$ holds precisely when $T$ is surjective.

Let $\mathcal{N}^{\infty}(T):=\bigcup_{k=1}^{\infty} \operatorname{ker} T^{k}$ and $\mathcal{R}^{\infty}(T):=\bigcap_{k=1}^{n} R\left(T^{k}\right)$ denote the hyper-kernel and the hyper-range of an operator $T \in L(X)$. For every $n \in \mathbb{N}$, we have the increasing chain of kernel-type subspaces

$$
\operatorname{ker} T \subseteq \operatorname{ker} T^{n} \subseteq \mathcal{N}^{\infty}(T) \subseteq H_{o}(T) \subseteq X_{T}(\{0\},
$$

and also the increasing chain of range-type subspaces

$$
X_{T}(\emptyset) \subseteq K(T) \subseteq \mathcal{R}^{\infty}(T) \subseteq R\left(T^{n}\right) \subseteq R(T) ;
$$

see [21, Propositions 1.2.16, 3.3.7, and 3.3.13]. By [1, Theorem 2.22 and Corollary 2.41] or by [7, Corollary 6], the localized SVEP may be characterized as follows. This result will be one of our principal tools.

Theorem 1.3. For every operator $T \in L(X)$ and $\lambda \in \mathbb{C}$, the following assertions are equivalent:

(i) $T$ has $S V E P$ at $\lambda$;

(ii) $\operatorname{ker}(\lambda I-T) \cap X_{T}(\emptyset)=\{0\}$;

(iii) $\mathcal{N}^{\infty}(\lambda I-T) \cap X_{T}(\emptyset)=\{0\}$.

\section{Results}

The interplay between Fredholm theory and the localized SVEP is of great interest. For instance, the localized SVEP at certain distinguished parts of the spectrum characterizes the classes of operators which satisfy Browder type theorems; see 3 . and [5]. For this reason, it is interesting to study the preservation of localized SVEP under certain perturbations.

We first explain why SVEP itself is not stable under arbitrary sums and products of commuting operators. While a specific example based on the theory of weighted shifts may be found in [11], here we present a general principle that shows that such examples exist in abundance. The following result is a slight extension of Corollary 4 of [24], but the present approach is considerably shorter. Note that, in Theorem 2.1 below, $T$ and $\exp (T)$ may fail to have SVEP, while the condition on $S$ entails that $X_{T}(\emptyset)=\{0\}$ and hence SVEP for $S$.

Theorem 2.1. Let $S, T \in L(X)$ be commuting operators, and suppose that there exist distinct points $\alpha, \beta \in \mathbb{C}$ for which

$$
K(S-\alpha I)=K(S-\beta I)=\{0\} .
$$

Then $T$ is the sum of two commuting operators with $S V E P$, while $\exp (T)$ is the product of two commuting operators with SVEP.

Proof. Since all quasi-nilpotent operators share SVEP, we may assume that $r(T)>$ 0 . To verify that $T(S-\alpha I)$ has SVEP, we consider an arbitrary open set $U \subseteq \mathbb{C}$ and an analytic function $f: U \rightarrow X$ for which $(\mu I-T(S-\alpha I)) f(\mu)=0$ for all $\mu \in U$. For fixed non-zero $\mu \in U$ and arbitrary $\lambda \in \mathbb{C}$ with $\lambda<|\mu| / r(T)$, the operator $\lambda T-\mu I$ is invertible and its inverse commutes with both $S$ and $T$. Moreover,

$$
\begin{aligned}
& (\lambda I-(S-\alpha I)) T(\lambda T-\mu I)^{-1} f(\mu) \\
= & (\lambda T-\mu I)^{-1}[(\mu I-T(S-\alpha I))+(\lambda T-\mu I)] f(\mu)=f(\mu) .
\end{aligned}
$$


This shows that $0 \in \rho_{S-\alpha I}(f(\mu))$ and therefore $f(\mu) \in K(S-\alpha I)=\{0\}$ for all non-zero $\mu \in U$. Thus $f \equiv 0$ on $U$, which establishes SVEP for $T(S-\alpha I)$ and, of course, similarly also for $T(S-\beta I)$. Because $(\beta-\alpha) T=T(S-\alpha I)+T(\beta I-S)$, the first assertion is now immediate, and the last claim follows from the fact that SVEP is preserved under the analytical calculus; see [21, Theorem 3.3.9].

It remains to provide concrete examples of operators that satisfy all the conditions of the preceding result. The condition on $S$ has been addressed in 21, Propositions 1.6.4 and 1.6.8]. In particular, if $S \in L(X)$ is a semi-shift, in the sense that $S$ is an isometry for which $\mathcal{R}^{\infty}(S)=\{0\}$, then $\sigma_{S}(x)$ coincides with the closed unit disc $D$ for all non-zero $x \in X$. This ensures that the identity $K(S-\alpha I)=\{0\}$ holds for arbitrary $\alpha \in D$.

To find an operator without SVEP that commutes with a semi-shift is perhaps not completely obvious, but this task can easily be accomplished when $X$ is a separable Hilbert space. Indeed, in this case, for arbitrary $S, T \in L(X)$ the operators $T \otimes I$ and $I \otimes S$ on the Hilbert tensor product $X \otimes X$ commute, since

$$
(T \otimes I)(I \otimes S)=T \otimes S=(I \otimes S)(T \otimes I) ;
$$

see [19, Section 2.6] for a nice exposition of the theory of the Hilbert tensor product. Moreover, since $T \otimes I$ is unitarily equivalent to the Hilbert direct sum $\sum_{n=1}^{\infty} \oplus T$, it is easily seen that the failure of SVEP at a point $\lambda$ extends from $T$ to $T \otimes I$. In the same vein, it follows that $I \otimes S$ is a semi-shift whenever $S$ is, since $I \otimes S$ is unitarily equivalent to $\sum_{n=1}^{\infty} \oplus S$. Note that, in the Hilbert spaces case, the semi-shifts are precisely pure isometries.

Thus neither SVEP nor localized SVEP is, in general, preserved under sums and products of commuting perturbations. On the positive side, if the operators $S$ and $T$ commute and share a property that is somewhat stronger than SVEP, namely Dunford's property $(C)$, then it is known from [21, Theorem 3.6.3] that $S+T$ has SVEP. Note that property $(C)$ simply means that $X_{T}(F)$ is closed for every closed subset $F$ of $\mathbb{C}$. Some other results on the preservation of SVEP may be found in 13 .

We now address the question of the extent to which SVEP at a point is stable under quasi-nilpotent equivalence. Recall from [12] and [21] that the commutator of two operators $S, T \in L(X)$ is the operator $C(S, T)$ on $L(X)$ given by

$$
C(S, T)(A):=S A-A T \text { for all } A \in L(X) .
$$

The operators $S$ and $T$ are said to be quasi-nilpotent equivalent provided that both $\|C(S, T)(I)\|^{1 / n} \rightarrow 0$ and $\|C(T, S)(I)\|^{1 / n} \rightarrow 0$ as $n \rightarrow \infty$. It is well known that quasi-nilpotent equivalent operators $S$ and $T$ satisfy the identity

$$
X_{T}(F)=X_{S}(F) \text { for all } F \subseteq \mathbb{C},
$$

and the latter condition actually characterizes quasi-nilpotent equivalence for pairs of decomposable operators; see, for instance, [21, Corollary 3.4.5]. If there exists an integer $n \in \mathbb{N}$ for which $C(S, T)^{n}(I)=C(T, S)^{n}(I)=0$, then the operators $S$ and $T$ are said to be nilpotent equivalent. For $S, T \in L(X)$ with $S T=T S$, it is easily seen that $C(S, T)^{n}(I)=(S-T)^{n}$ for all $n \in \mathbb{N}$. Thus, in this case, $S$ and $T$ are quasi-nilpotent equivalent precisely when $S-T$ is quasi-nilpotent, while $S$ and $T$ are nilpotent equivalent if and only if $S-T$ is nilpotent. 
Theorem 2.2. Suppose that the operators $S, T \in L(X)$ are nilpotent equivalent, and let $\lambda \in \mathbb{C}$. Then $T$ has SVEP at $\lambda$ precisely when $S$ does. In particular, if $T$ has $S V E P$ at $\lambda$, and if $N \in L(X)$ is nilpotent and satisfies $T N=N T$, then also $T+N$ has $S V E P$ at $\lambda$.

Proof. By symmetry it suffices to show that SVEP at $\lambda$ is transferred from $S$ to $T$. By Theorem 1.3 the condition on $S$ entails that

$$
\mathcal{N}^{\infty}(\lambda I-S) \cap X_{S}(\emptyset)=\{0\},
$$

while the nilpotent equivalence of $S$ and $T$ ensures that $X_{S}(\emptyset)=X_{T}(\emptyset)$. Moreover, straightforward induction shows that

$$
\begin{aligned}
C(S, T)^{n}(A) & =(-1)^{n} C(\lambda I-S, \lambda I-T)^{n}(A) \\
& =\sum_{k=0}^{n}\left(\begin{array}{c}
n \\
k
\end{array}\right)(-1)^{n-k}(\lambda I-S)^{n-k} A(\lambda I-T)^{k}
\end{aligned}
$$

for all $A \in L(X)$ and $n \in \mathbb{N}$. We now choose an $n \in \mathbb{N}$ for which $C(S, T)^{n}(I)=0$ and consider an arbitrary $x \in \operatorname{ker}(\lambda I-T)$. Then $(\lambda I-T)^{k} x=0$ for $k=1, \ldots, n$, so that the preceding identities imply that $(\lambda I-S)^{n} x=0$. Consequently, we obtain

$$
\operatorname{ker}(\lambda I-T) \subseteq \operatorname{ker}(\lambda I-S)^{n} \subseteq \mathcal{N}^{\infty}(\lambda I-S)
$$

and therefore

$$
\operatorname{ker}(\lambda I-T) \cap X_{T}(\emptyset) \subseteq N^{\infty}(\lambda I-S) \cap X_{S}(\emptyset)=\{0\} .
$$

Hence Theorem 1.3 guarantees that $T$ has SVEP at $\lambda$.

Nilpotent operators are a special case of algebraic operators. Recall that an operator $K \in L(X)$ is said to be algebraic if there exists a non-trivial complex polynomial $h$ such that $h(K)=0$. In addition to nilpotent operators, examples of algebraic operators are idempotent operators and operators for which some power has finite-dimensional range. If $T \in L(X)$ has SVEP at a point $\lambda$, then it may be tempting to conjecture that $T+K$ has SVEP at $\lambda$ for every algebraic operator $K$ that commutes with $T$. However, this cannot be true in general, since SVEP for $T$ at $\lambda$ is equivalent to SVEP for $T-\lambda I$ at 0 . Nevertheless, we obtain the following result.

Theorem 2.3. Suppose that $T, K \in L(X)$ are commuting operators, suppose that $K$ is algebraic, and let $h$ be a non-zero polynomial for which $h(K)=0$. If $T$ has $S V E P$ at each of the zeros of $H$, then $T-K$ has $S V E P$ at 0 . In particular, if $T$ has $S V E P$, then so does $T+K$.

Proof. By the classical spectral mapping theorem, $h(\sigma(K))=\sigma(h(K))=\{0\}$, so that $\sigma(K)$ is finite, say $\sigma(K)=\left\{\mu_{1}, \ldots, \mu_{n}\right\}$. For $i=1, \ldots, n$ let $P_{i} \in L(X)$ denote the spectral projection associated with $K$ and with the spectral set $\left\{\mu_{i}\right\}$, and let $Y_{i}:=R\left(P_{i}\right)$. From standard spectral theory it is known that $P_{1}+\cdots+P_{n}=I$, that $Y_{1}, \ldots, Y_{n}$ are closed linear subspaces of $X$ which are each invariant under both $K$ and $T$, and that $X=Y_{1} \oplus \cdots \oplus Y_{n}$. Moreover, for arbitrary $i=1, \ldots, n$, the two restrictions $K_{i}:=K \mid Y_{i}$ and $T_{i}:=T \mid Y_{i}$ commute, and we have $\sigma\left(K_{i}\right)=\left\{\mu_{i}\right\}$. Because $h\left(K_{i}\right)=h(K) \mid Y_{i}=0$, we obtain

$$
h\left(\left\{\mu_{i}\right\}\right)=h\left(\sigma\left(K_{i}\right)\right)=\sigma\left(h\left(K_{i}\right)\right)=\{0\} .
$$


Hence we may factor $h$ in the form

$$
h(\mu)=\left(\mu-\mu_{i}\right)^{n_{i}} q_{i}(\mu) \text { for all } \mu \in \mathbb{C},
$$

where $n_{i} \in \mathbb{N}$ and $q_{i}$ is a complex polynomial for which $q_{i}\left(\mu_{i}\right) \neq 0$. We conclude that

$$
0=h\left(K_{i}\right)=\left(K_{i}-\mu_{i} I\right)^{n_{i}} q_{i}\left(K_{i}\right),
$$

where $q_{i}\left(K_{i}\right) \in L\left(Y_{i}\right)$ is invertible in light of $\sigma\left(q_{i}\left(K_{i}\right)\right)=q_{i}\left(\sigma\left(K_{i}\right)\right)=\left\{q_{i}\left(\mu_{i}\right)\right\}$ and $q_{i}\left(\mu_{i}\right) \neq 0$. Therefore $\left(K_{i}-\mu_{i} I\right)^{n_{i}}=0$, which shows that the operator $N_{i}:=$ $K_{i}-\mu_{i} I$ is nilpotent. Now observe that

$$
T_{i}-K_{i}=\left(T_{i}-\mu_{i} I\right)-\left(K_{i}-\mu_{i} I\right)=T_{i}-\mu_{i} I-N_{i} .
$$

Because $T$ has SVEP at $\mu_{i}$, we know that $T-\mu_{i} I$ has SVEP at 0 . Since this condition is inherited by restrictions to closed invariant subspaces, we conclude that $T_{i}-\mu_{i} I$ has SVEP at 0, and hence, by Theorem 2.2. also $T_{i}-K_{i}=T_{i}-\mu_{i} I-N_{i}$ has SVEP at 0 for all $i=1, \ldots, n$. By [1, Theorem 2.9], it then follows that

$$
T-K=\left(T_{1}-K_{1}\right) \oplus \cdots \oplus\left(T_{n}-K_{n}\right)
$$

has SVEP at 0 , as desired. An application of the main result to the operator $-K$ and $T-\lambda I$ for arbitrary $\lambda \in \mathbb{C}$ then establishes the final claim.

We mention that the last assertion of Theorem 2.3 ceases to be true for noncommuting operators. In fact, by [26. Example 5.6.29], the sum of a decomposable operator and a rank-one operator may fail to have SVEP, although decomposable operators have SVEP and rank-one operators are algebraic.

The case of commuting quasi-nilpotent perturbations seems to be more complicated. Since an operator $T \in L(X)$ has SVEP precisely when $X_{T}(\emptyset)=\{0\}$, and since quasi-nilpotent equivalence preserves the analytic spectral subspaces, it is clear that SVEP is stable under quasi-nilpotent equivalence. The next result may be viewed as a certain localized version of this fact. In Theorem 2.4, we assume that $H_{0}(\lambda I-T) \cap X_{T}(\emptyset)=\{0\}$. Because $\operatorname{ker}(\lambda I-T) \subseteq H_{0}(\lambda I-T)$, this condition entails, by Theorem 1.3. that $T$ has SVEP at $\lambda$.

Theorem 2.4. Suppose that $T \in L(X)$ satisfies $H_{0}(\lambda I-T) \cap X_{T}(\emptyset)=\{0\}$ for some $\lambda \in \mathbb{C}$, and let $S \in L(X)$ be quasi-nilpotent equivalent to $T$. Then $S$ has $S V E P$ at $\lambda$. In particular, $T+Q$ has $S V E P$ at $\lambda$ for every quasi-nilpotent operator $Q \in L(X)$ for which $T Q=Q T$.

Proof. Let $x \in \operatorname{ker}(\lambda I-S)$. Then $(\lambda I-S)^{k} x=0$ for all $k \in \mathbb{N}$. Moreover, for arbitrary $n \in \mathbb{N}$, we know from the proof of Theorem 2.2 that

$$
C(T, S)^{n}(I)=\sum_{k=0}^{n}\left(\begin{array}{c}
n \\
k
\end{array}\right)(-1)^{n-k}(\lambda I-T)^{n-k}(\lambda I-S)^{k} .
$$

Consequently, we obtain that

$$
\left\|(\lambda I-T)^{n} x\right\|^{1 / n}=\left\|C(T, S)^{n}(I) x\right\|^{1 / n} \leq\left\|C(T, S)^{n}(I)\right\|^{1 / n}\|x\|^{1 / n} \rightarrow 0
$$

as $n \rightarrow \infty$. Thus $\operatorname{ker}(\lambda I-S) \subseteq H_{0}(\lambda I-T)$, while $X_{S}(\emptyset)=X_{T}(\emptyset)$, by quasinilpotent equivalence. We conclude that

$$
\operatorname{ker}(\lambda I-S) \cap X_{S}(\emptyset) \subseteq H_{0}(\lambda I-T) \cap X_{T}(\emptyset)=\{0\},
$$

so that Theorem 1.3 ensures that $S$ has SVEP at $\lambda$. 
Corollary 2.5. Suppose that $T \in L(X)$ and $\lambda \in \mathbb{C}$ satisfy the condition that $H_{0}(\lambda I-T) \cap K(\lambda I-T)=\{0\}$ or, more generally, that $H_{0}(\lambda I-T) \cap K(\lambda I-T)$ is closed. Then every operator that is quasi-nilpotent equivalent to T has SVEP at $\lambda$.

Proof. By [7, Corollary], the two conditions on $H_{0}(\lambda I-T) \cap K(\lambda I-T)$ are actually equivalent. Because $X_{T}(\emptyset) \subseteq K(\lambda I-T)$, in either case we conclude that $H_{0}(\lambda I-T) \cap X_{T}(\emptyset)=\{0\}$, so Theorem 2.4 applies.

Question. Is SVEP at a point preserved under quasi-nilpotent commuting perturbations or even under quasi-nilpotent equivalence?

Although we do not know the answer to this question in general, we can handle certain important special cases. For simplicity, for this we focus on the case $\lambda=0$. Because $\mathcal{N}^{\infty}(T) \cap X_{T}(\emptyset) \subseteq H_{0}(T) \cap K(T)$, it is immediate from Theorem 1.3 that the condition $H_{0}(T) \cap K(T)=\{0\}$ entails SVEP for $T$ at 0 . A counterexample based on the theory of weighted shifts on a Hilbert space of certain formal Laurent series shows that the converse is not true in general; see [7, Example 1].

The condition $H_{0}(T) \cap K(T)=\{0\}$ has been studied in several papers [2, 6], [7, [9], for certain classes of operators that naturally arise in Fredholm theory. We briefly review the relevant notions that will be used in the sequel.

For arbitrary $T \in L(X)$, let $\alpha(T):=\operatorname{dim} \operatorname{ker} T$ and $\beta(T):=\operatorname{codim} R(T)$. As usual,

$$
\Phi_{+}(X):=\{T \in L(X): \alpha(T)<\infty \text { and } T(X) \text { is closed }\}
$$

denotes the class of upper semi-Fredholm operators, while

$$
\Phi_{-}(X):=\{T \in L(X): \beta(T)<\infty\}
$$

stands for the class of lower semi-Fredholm operators, and $\Phi_{+}(X) \cup \Phi_{-}(X)$ is the class of semi-Fredholm operators on $X$.

Also recall that an operator $T \in L(X)$ is said to be semi-regular if $R(T)$ is closed and $\mathcal{N}^{\infty}(T) \subseteq \mathcal{R}^{\infty}(T)$. More generally, $T$ is said to admit a generalized Kato decomposition if there exists a pair $(M, N)$ of $T$-invariant closed subspaces of $X$ such that $X=M \oplus N, T \mid M$ is semi-regular, and $T \mid N$ is quasi-nilpotent. If, in addition, $N$ is of finite dimension, then $T$ is said to be essentially semi-regular; see [1. Chapter 1] for details and properties of such operators. Observe that semiFredholm operators are essentially semi-regular, but need not be semi-regular; see [1. Theorems 1.58 and 1.62].

For the definition of quasi-Fredholm operators, we need the notation

$$
\Delta(T):=\left\{n \in \mathbb{N}: R\left(T^{n}\right) \cap \operatorname{ker} T \subseteq R\left(T^{m}\right) \cap \text { ker } T \text { for } m \in \mathbb{N} \text {, with } m \geq n\right\} .
$$

The degree of stable iteration is defined as $\operatorname{dis}(T):=\inf \Delta(T)$ if $\Delta(T) \neq \emptyset$, while $\operatorname{dis}(T)=\infty$ otherwise.

Definition 2.6. $T \in L(X)$ is said to be quasi-Fredholm provided that $d:=\operatorname{dis}(T)$ is finite and the spaces $R(T)+\operatorname{ker} T^{d}$ and $R\left(T^{n}\right)$ for arbitrary $n \geq d$ are closed.

The class of all quasi-Fredholm operators on $X$ is denoted by $Q F(X)$. In the Hilbert space setting, the investigation of such operators dates back, in an equivalent form, to Labrousse [20, Definition 3.1.2], while Mbekhta and Müller [23] were the first to study such operators on Banach spaces. For a discussion of the relationship between the localized SVEP and quasi-Fredholm operators, we refer to [2]. 
There are also interesting connections to the notion of Drazin invertibility. More precisely, if $T \in L(X)$ is left Drazin invertible, in the sense that $p:=p(T)$ is finite and $R\left(T^{p+1}\right)$ is closed, then $T$ is quasi-Fredholm. Similarly, if $T$ is right Drazin invertible, in the sense that $q:=q(T)$ is finite and $R\left(T^{q}\right)$ is closed, then $T$ is quasi-Fredholm. In fact both statements easily follow from [23, Lemma 12] and an inspection of the quantities $k_{n}(T)$ introduced in [23.

An illuminating alternative approach may be based on the theory of semi- $B$ Fredholm operators, defined as those operators $T \in L(X)$ for which there exists some $n \in \mathbb{N}$ such that $R\left(T^{n}\right)$ is closed and the restriction of $T$ to $R\left(T^{n}\right)$ is semiFredholm. Indeed, by [10, Proposition 2.5], all semi $B$-Fredholm operators are quasi-Fredholm, while, by [4, Theorem 2.5], the left Drazin invertible operators are exactly the semi $B$-Fredholm operators that have SVEP at 0 , and the right Drazin invertible operators are precisely the semi $B$-Fredholm operators for which the adjoint has SVEP at 0. Furthermore, by [2, Theorem 2.7], we conclude that the left Drazin invertible operators coincide with the quasi-Fredholm operators that have SVEP at 0, while, by [2, Theorem 2.11], the right Drazin invertible operators are exactly the quasi-Fredholm operators for which the adjoint has SVEP at 0.

Theorem 2.7. Let $S, T \in L(X)$ be quasi-nilpotent equivalent operators, let $\lambda \in \mathbb{C}$ and suppose that $\lambda I-T$ either admits a generalized Kato decomposition or is quasiFredholm. If $T$ satisfies $S V E P$ at $\lambda$, then so does $S$.

Proof. Under either of the two conditions on $\lambda I-T$, it is known that SVEP for $T$ at $\lambda$ is equivalent to the condition $H_{0}(\lambda I-T) \cap K(\lambda I-T)=\{0\}$; see [6. Theorem 2.7] and [2, Theorem 2.7]. Consequently, the assertion is clear from Corollary 2.5.

We now turn to the preservation of the localized SVEP under commuting Riesz perturbations. Recall that an operator $T \in L(X)$ is said to be a Riesz operator provided that $\lambda I-T \in \Phi_{+}(X) \cap \Phi_{-}(X)$, for all $\lambda \in \mathbb{C} \backslash\{0\}$. In addition to the quasinilpotent operators, the class of Riesz operators contains all compact operators.

Also recall that an operator $T \in L(X)$ is said to be upper semi-Browder if $T \in \Phi_{+}(X)$ and $p(T)<\infty$, while an operator $T \in L(X)$ for which $T \in \Phi_{-}(X)$ and $q(T)<\infty$ is said to be lower semi-Browder. By a result of Rakočević ([25]), the semi-Browder operators are stable under Riesz commuting perturbations; i.e., if $T \in L(X)$ and $R \in L(X)$ is a Riesz operator for which $R T=T R$, then

$T$ is upper semi-Browder $\Leftrightarrow T+R$ is upper semi-Browder,

and also

$T$ is lower semi-Browder $\Leftrightarrow T+R$ is lower semi-Browder.

Very few results are known about the permanence of SVEP under commuting compact perturbations. In [28] the stability of SVEP under small compact perturbations has been studied for operators acting on separable Hilbert spaces. The following theorem now shows that the localized SVEP is preserved under arbitrary commuting Riesz perturbations, in particular, in the semi-Fredholm case.

Theorem 2.8. Let $T \in L(X)$, and suppose that $R \in L(X)$ is a Riesz operator that commutes with $T$. Then, for each $\lambda \in \mathbb{C}$ for which $\lambda I-T$ is essentially semi-regular, the following assertions hold:

(i) If $T$ has SVEP at $\lambda$, then $T+R$ has $S V E P$ at $\lambda$.

(ii) If $T^{*}$ has SVEP at $\lambda$, then $T^{*}+R^{*}$ has $S V E P$ at $\lambda$. 
Proof. We first show that an operator $T \in L(X)$ is essentially semi-regular and satisfies SVEP at 0 precisely when $T$ is upper semi-Browder. Indeed, if $T$ is upper semi-Browder, then (11) ensures that $T$ has SVEP at 0 , while we know from [1, Theorem 1.62] that $T$ is essentially semi-regular. Conversely, suppose that $T$ satisfies the latter conditions, and consider a pair of $T$-invariant closed subspaces $M$ and $N$ of $X$ such that $X=M \oplus N, N$ is finite dimensional, $T \mid M$ is semi-regular, and $T \mid N$ is quasi-nilpotent. Because $\operatorname{dim} N<\infty$, it follows that $T \mid N$ is actually nilpotent. Also, by [1, Theorem 3.14], SVEP for $T$ at 0 ensures that $N=H_{0}(T)$ and therefore $\alpha(T)<\infty$, because ker $T \subseteq H_{0}(T)$ and $\operatorname{dim} N<\infty$. Moreover,

$$
T(X)=T(M) \oplus T(N),
$$

where $T(M)$ is closed, since $T \mid M$ is semi-regular, and $T(N)$ is of finite dimension. Thus $T$ has closed range and hence is upper semi-Fredholm. Moreover, by [1, Theorem 3.16], SVEP for $T$ at 0 is equivalent to the condition $p(T)<\infty$. Thus $T$ is upper semi-Browder, as desired.

Assertion (i) is now easily established. Indeed, if $\lambda I-T$ is essentially semiregular and $T$ has SVEP at $\lambda$, then we know that $\lambda I-T$ is upper semi-Browder. By (5), it follows that $\lambda I-T-R$ is upper semi-Browder and hence $T+R$ has SVEP at $\lambda$, again by the characterization of the preceding paragraph.

Finally, assertion (ii) follows from (i) by duality. Indeed, it suffices to observe that, by [1, Corollary 1.49], $T$ is essentially semi-regular precisely when $T^{*}$ satisfies this condition, while standard Fredholm theory entails that $R$ is a Riesz operator if and only if $R^{*}$ is.

To establish a certain counterpart of Theorem 2.8 for quasi-Fredholm operators, we introduce the classes

$$
Q F_{+}(X):=\{T \in Q F(X): \alpha(T)<\infty\}
$$

and

$$
Q F_{-}(X):=\{T \in Q F(X): \beta(T)<\infty\} .
$$

Theorem 2.9. Let $T \in L(X)$ and suppose that $R \in L(X)$ is a Riesz operator which commutes with $T$. Then, for each $\lambda \in \mathbb{C}$, the following assertions hold:

(i) If $\lambda I-T \in Q F_{+}(X)$ and $T$ has SVEP at $\lambda$, then $T+R$ has SVEP at $\lambda$.

(ii) If $\lambda I-T \in Q F_{-}(X)$ and $T^{*}$ has SVEP at $\lambda$, then $T^{*}+R^{*}$ has SVEP at $\lambda$.

Proof. (i) We can assume $\lambda=0$. Observe first that if $T$ is quasi-Fredholm, then SVEP for $T$ at 0 is equivalent to the existence of some $n \in \mathbb{N}$ for which $T^{n}(X)$ is closed and the restriction $T \mid T^{n}(X)$ is bounded below; see [2, Theorem 2.7]. Moreover, it is an elementary fact that $\alpha(T)<\infty$ entails that $\alpha\left(T^{n}\right)<\infty$, hence $T^{n} \in \Phi_{+}(X)$, and from classical Fredholm theory we conclude that also $T \in \Phi_{+}(X)$. SVEP for $T+R$ at 0 then follows from part (i) of Theorem 2.8 .

(ii) This assertion follows from (i) by duality, but there is also an independent short argument. Again, we may assume $\lambda=0$. If $T$ is quasi-Fredholm, then, by [2, Theorem 2.11], SVEP for $T^{*}$ at 0 is equivalent to the existence of some $n \in \mathbb{N}$ for which $T^{n}(X)$ is closed and $T \mid T^{n}(X)$ is surjective. Since $\beta(T)<\infty$ implies that $\beta\left(T^{n}\right)<\infty$, we infer that $T^{n} \in \Phi_{-}(X)$ and hence that also $T \in \Phi_{-}(X)$, again by the classical Fredholm theory. SVEP for $T^{*}+R^{*}$ at 0 is then immediate from part (ii) of Theorem 2.8 
We finally address the permanence of localized SVEP for the adjoint $T^{*}$ of an operator $T \in L(X)$. The condition $H_{0}(\lambda I-T)+K(\lambda I-T)=X$ may be thought of as being dual to the condition $H_{0}(\lambda I-T) \cap K(\lambda I-T)=\{0\}$ and entails SVEP for $T^{*}$ at $\lambda$. In fact, this is immediate from (3) and the characterization

$$
H_{0}(\lambda I-T)+K(\lambda I-T)=X \Leftrightarrow \sigma_{\mathrm{s}}(T) \text { does not cluster at } \lambda .
$$

The preceding equivalence is obvious if $\lambda \notin \sigma_{\mathrm{s}}(T)$, since $K(\lambda I-T)=X$ in this case. On the other hand, if $\lambda \in \sigma_{\mathrm{s}}(T)$, then, by [18, Theorem 5], the identity $H_{0}(\lambda I-T)+K(\lambda I-T)=X$ holds precisely when $\lambda$ is an isolated point of $\sigma_{\mathrm{s}}(T)$. Moreover, since $\sigma_{\mathrm{s}}\left(T^{*}\right)=\sigma_{\mathrm{a}}(T)$, we obtain the characterization

$$
H_{0}\left(\lambda I-T^{*}\right)+K\left(\lambda I-T^{*}\right)=X^{*} \Leftrightarrow \sigma_{\mathrm{a}}(T) \text { does not cluster at } \lambda .
$$

In particular, by $(2)$ the condition $H_{0}\left(\lambda I-T^{*}\right)+K\left(\lambda I-T^{*}\right)=X^{*}$ implies SVEP for $T$ at $\lambda$. These observations concerning the localized SVEP will be improved in the following result.

Recall that the annihilator of a linear subspace $M$ of $X$ is defined as

$$
M^{\perp}:=\left\{\phi \in X^{*}: \phi(x)=0 \text { for all } x \in M\right\},
$$

while the pre-annihilator of a linear subspace $N$ of $X^{*}$ is defined as

$$
{ }^{\perp} N:=\{x \in X: \phi(x)=0 \text { for all } \phi \in N\} .
$$

By the bipolar theorem ${ }^{\perp}\left(M^{\perp}\right)$ is the norm closure of $M$, and $\left.\left({ }^{\perp} N\right)^{\perp}\right)$ is the weak*-closure of $N$.

Theorem 2.10. For every pair of quasi-nilpotent equivalent operators $S, T \in L(X)$ and arbitrary $\lambda \in \mathbb{C}$, the following assertions hold:

(i) if $K(\lambda I-T)+H_{0}(\lambda I-T)$ is norm dense in $X$, then $S^{*}$ has $S V E P$ at $\lambda$.

(ii) if $H_{0}\left(\lambda I-T^{*}\right)+K\left(\lambda I-T^{*}\right)$ is weak-*-dense in $X^{*}$, then $S$ has SVEP at $\lambda$.

Proof. (i) By [1, Theorem 1.70], we have the inclusions

$$
H_{0}(\lambda I-T) \subseteq{ }^{\perp} K\left(\lambda I-T^{*}\right) \text { and } K(\lambda I-T) \subseteq{ }^{\perp} H_{0}\left(\lambda I-T^{*}\right),
$$

and therefore, by duality,

$$
K\left(\lambda I-T^{*}\right) \subseteq H_{0}\left(\lambda I-T^{*}\right)^{\perp} \quad \text { and } \quad H_{0}\left(\lambda I-T^{*}\right) \subseteq K(\lambda I-T)^{\perp} .
$$

We conclude that

$$
\begin{aligned}
K\left(\lambda I-T^{*}\right) \cap H_{0}\left(\lambda I-T^{*}\right) & \subseteq H_{0}(\lambda I-T)^{\perp} \cap K(\lambda I-T)^{\perp} \\
& =\left[H_{0}(\lambda I-T)+K(\lambda I-T)\right]^{\perp}=\{0\},
\end{aligned}
$$

where the last equality follows from the condition that $H_{0}(\lambda I-T)+K(\lambda I-T)$ is norm dense in $X$. Moreover, since

$$
[C(S, T)(A)]^{*}=(-1)^{n} C\left(T^{*}, S^{*}\right)^{n}\left(A^{*}\right)
$$

for all $A \in L(X)$ and $n \in \mathbb{N}$, it is clear that the pair $\left(S^{*}, T^{*}\right)$ inherits quasinilpotent equivalence from the pair $(S, T)$. The assertion is now immediate from Corollary 2.5.

(ii) Similarly, we obtain

$$
\begin{aligned}
H_{0}(\lambda I-T) \cap K(\lambda I-T) & \subseteq{ }^{\perp} K\left(\lambda I-T^{*}\right) \cap{ }^{\perp} H_{0}\left(\lambda I-T^{*}\right) \\
& ={ }^{\perp}\left[K\left(\lambda I-T^{*}\right)+H_{0}\left(\lambda I-T^{*}\right)\right]=\{0\},
\end{aligned}
$$


where the last identity follows from the Hahn-Banach theorem and the weak*density of $H_{0}\left(\lambda I-T^{*}\right)+K\left(\lambda I-T^{*}\right)$ in $X^{*}$. Another application of Corollary 2.5 now ensures that $S$ has SVEP at $\lambda$.

Note that Theorem 2.10 improves [7, Corollary 8], where the assertions (i) and (ii) of the preceding result were established in the perturbation-free case $S=T$. Moreover, if the operator $\lambda I-T$ admits a generalized Kato decomposition, then the condition considered in (i) admits a simple interpretation. Indeed, in this case, 1, Theorem 3.15] ensures that $T^{*}$ has SVEP at $\lambda$ precisely when $H_{0}(\lambda I-T)+K(\lambda I-T)$ is norm dense in $X$, and that this happens if and only if $H_{0}(\lambda I-T)+K(\lambda I-T)=X$.

\section{REFERENCES}

[1] P. Aiena, Fredholm and local spectral theory, with application to multipliers. Kluwer Acad. Publishers, 2004. MR2070395 (2005e:47001)

[2] P. Aiena, Quasi-Fredholm operators and localized SVEP, Acta Sci. Math. (Szeged) 73 (2007), 251-263. MR2339864 (2008g:47014)

[3] P. Aiena, M. T. Biondi, Browder's theorem through localized SVEP. Mediterranean J. of Math. 2 (2005), 137-151. MR2184191 (2006h:47003)

[4] P. Aiena, M. T. Biondi, C. Carpintero, On Drazin invertibility. Proc. Amer. Math. Soc. 136 (2008), 2839-2848. MR2399049 (2009b:47001)

[5] P. Aiena, C. Carpintero, E. Rosas, Some characterizations of operators satisfying a-Browder's theorem, J. Math. Anal. Appl. 311 (2005), 530-544. MR2168416 (2006e:47005)

[6] P. Aiena, M. L. Colasante, M. González, Operators which have a closed quasi-nilpotent part. Proc. Amer. Math. Soc. 130 (2002), 2701-2710. MR.1900878 (2003g:47008)

[7] P. Aiena, L. Miller, M.M. Neumann, On a localised single-valued extension property, Math. Proc. R. Ir. Acad. 104A (2004), no. 1, 17-34. MR2139507 (2005k:47011)

[8] P. Aiena, O. Monsalve, Operators which do not have the single valued extension property. J. Math. Anal. Appl. 250 (2000), 435-448. MR.1786074 (2001g:47005)

[9] P. Aiena, O. Monsalve, The single valued extension property and the generalized Kato decomposition property. Acta Sci. Math. (Szeged) 67 (2001), 791-807. MR1876467 (2002i:47018)

[10] M. Berkani, M. Sarih, On semi B-Fredholm operators, Glasgow Math. J. 43 (2001), 457-465. MR.1878588 (2002j:47017)

[11] A. Bourhim, V. G. Miller, The single-valued extension property is not preserved under sums and products of commuting operators. Glasgow Math. J. 49 (2007), 99-104. MR.2337870 (2008h:47012)

[12] I. Colojoară, C. Foiaş, Theory of generalized spectral operators. Gordon and Breach, New York, 1968. MR0394282 (52:15085)

[13] D. Drissi, The single-valued extension property and subharmonicity. Extracta Math. 22 (2007), no. 3, 235-244. MR2405083 (2008m:47003)

[14] N. Dunford, Spectral theory II. Resolution of the identity. Pacific J. Math. 2 (1952), 559-614. MR0051435 (14:479a)

[15] N. Dunford, Spectral operators. Pacific J. Math. 4 (1954), 321-354. MR0063563 (16:142d)

[16] N. Dunford, J. T. Schwartz, Linear operators, Part III: Spectral operators. Wiley, New York, 1971. MR0412888(54:1009)

[17] J. K. Finch, The single valued extension property on a Banach space, Pacific J. Math. 58 (1975), 61-69. MR0374985(51:11181)

[18] M. González, M. Mbekhta, M. Oudghiri, On the isolated points of the surjective spectrum of a bounded operator, Proc. Amer. Math. Soc. 136 (2008), no. 10, 3521-3528. MR2415036 (2009i:47005)

[19] R. V. Kadison, J. R. Ringrose, Fundamentals of the theory of operator algebras, Vol. I, Academic Press, New York, 1983. MR719020 (85j:46099)

[20] J. P. Labrousse, Les opérateurs quasi-Fredholm: une généralisation des opérateurs semiFredholm, Rend. Circ. Mat. Palermo (2) 29 (1980), 161-258. MR636072 (83c:47022)

[21] K. B. Laursen, M. M. Neumann, Introduction to local spectral theory, Clarendon Press, Oxford, 2000. MR1747914(2001k:47002) 
[22] M. Mbekhta, Sur la théorie spectrale locale et limite des nilpotents, Proc. Amer. Math. Soc. 110 (1990), 621-631. MR 1004421 (91b:47004)

[23] M. Mbekhta, V. Müller, On the axiomatic theory of spectrum II, Studia Math. 119 (1996), 129-147. MR1391472 (97c:47005)

[24] T. L. Miller, M. M. Neumann, The single-valued extension property for sums and products of commuting operators. Czechoslovak Math. J. 52(127) (2002), no. 3, 635-642. MR1923267 (2003e:47006)

[25] V. Rakočević, Semi-Browder operators and perturbations. Studia Math. 122 (1997), 131-137. MR 1432164 (98g:47010)

[26] F. H. Vasilescu, Analytic functional calculus and spectral decompositions. Editura Academiei and D. Reidel Publishing Company, Bucharest and Dordrecht, 1982. MR690957 (85b:47016)

[27] P. Vrbová, On local spectral properties of operators in Banach spaces, Czechoslovak Math. J. 23(98) (1973a), 483-92. MR0322536 (48:898)

[28] S. Zhu, C. Li, SVEP and compact perturbations, J. Math. Anal. Appl. 380 (2011), 69-75. $\operatorname{MR} 2786184$

Dipartimento di Metodi e Modelli Matematici, Facoltà di Ingegneria, Università di Palermo, Viale delle Scienze, I-90128 Palermo, Italy

E-mail address: paiena@unipa.it

Department of Mathematics and Statistics, Mississippi State University, Mississippi StATE, Mississippi 39762

E-mail address: neumann@math.msstate.edu 